\title{
Dynamic Shear Modulus of Tricresyl Phosphate and Squalane
}

\author{
Robert D. Deegan, Robert L. Leheny, Narayanan Menon, and Sidney R. Nagel* \\ The James Franck Institute and The Department of Physics, The University of Chicago, \\ Chicago, Illinois 60637 \\ David C. Venerus \\ Department of Chemical Engineering, Illinois Institute of Technology, Chicago, Illinois 60616
}

Received: September 23, 1998; In Final Form: January 11, 1999

\begin{abstract}
We have measured the shear modulus and viscosity of two supercooled liquids: tricresyl phosphate and squalane. In both cases we find that the width of the peak in the imaginary part of the shear modulus narrows as the temperature is lowered toward the glass transition temperature. For tricresyl phosphate, we find no evidence for a decoupling of the viscosity and shear modulus relaxation times from the relaxation times determined by dielectric spectroscopy.
\end{abstract}

As a liquid is supercooled toward its glass transition temperature, its inherent relaxation times increase and its viscosity rises. ${ }^{1}$ For many years it was commonly accepted that the viscosity, $\eta$, was simply proportional to the relaxation time, $\tau$, as for example measured by dielectric response or light scattering. Such a relationship would be suggested by the Einstein-Debye equation ${ }^{2}$

$$
\eta=\left(k_{\mathrm{B}} T / V\right) \tau
$$

where $k_{\mathrm{B}}$ is Boltzmann's constant, $T$ is the temperature, and $V$ is the molecular volume. The proportionality between $\eta$ and $\tau$ is also a consequence of the Maxwell model ${ }^{3}$ for viscoelastic liquids, which gives the constant of proportionality as the infinite frequency shear modulus, $G_{\infty}$ :

$$
\eta=G_{\infty} \tau
$$

Thus, it was assumed that both $\eta$ and $\tau$ would increase in the same manner as the temperature of the liquid was lowered.

Angell ${ }^{4}$ pointed out, however, that such a relationship is by no means guaranteed and suggested the exciting possibility that it might be violated in supercooled liquids if certain relaxation processes decouple from the viscosity. This idea has been taken up by several groups, ${ }^{5-7}$ and some experiments have reported evidence of deviations from strict proportionality. In one such experiment, ${ }^{7}$ the deviations were suggested as providing a measurement of a diverging length scale in the cooperative dynamics as the glass transition temperature, $T_{\mathrm{g}}$, was approached.

Measurements of the real and imaginary parts of the dynamic shear modulus, $G(v)=G^{\prime}(v)+\mathrm{i} G^{\prime \prime}(v)$, can determine directly the time scale that appears in shear relaxation. However, such measurements on simple (i.e., low molecular weight) liquids are not widely available. ${ }^{8}$ A previous measurement on di- $n$ butylphthalate ${ }^{9}$ indicated that the time scales measured from $G(v)$ followed the same behavior as that measured in dielectric spectroscopy. However, it was found that the width of the shear modulus response became narrower on lowering the temperature

* To whom correspondence should be addressed. rather than wider as is commonly found in the dielectric response of nonionic glass formers. To test the generality of the results presented on that liquid, we here present measurements of $G(v)$ on two other glass-forming liquids: tricresyl phosphate (obtained from Alfa Aesar with a 50/50 mixture of para and meta and less than $1 \%$ ortho isomers) and squalane (obtained from Aldrich Chemical). These two glass formers are fragile, whereas di- $n$ butylphthalate is intermediate, in the Angell classification scheme for supercooled liquids. ${ }^{10}$

Our procedure for measuring the shear modulus was reported previously. ${ }^{9}$ We used a Rheometrics RMS 800 mechanical spectrometer in which the sample was contained between two parallel circular plates. The strain, $\gamma$, was produced by rotating the bottom plate while the stress, $\sigma$, was determined from the torque necessary to hold the top plate motionless. We measured the dynamic shear modulus, $G(v) \equiv \sigma_{\nu}(t) / \gamma_{\nu}(t)$, by applying an oscillatory strain, $\gamma_{v}(t)=\gamma \mathrm{e}^{\mathrm{i} 2 \pi v t}$ and measuring the resultant stress, $\sigma_{v}(t)=\sigma \mathrm{e}^{\mathrm{i}(2 \pi v t+\phi)}$. The amplitude $\gamma$ could be varied and was kept small to ensure linear response. The torque produced at a given driving frequency, $\nu$, and oscillation amplitude, $\gamma$, by a low-molecular-weight liquid is much larger than it is by a polymeric system in the same geometry. One reason for the paucity of $G(v)$ data on the simple liquids is that many machines designed to measure the shear modulus were specifically intended to study "softer" polymer liquids. To counter this problem, we machined small transducer plates, down to $1 \mathrm{~mm}$ in radius, to hold the sample so that the torque produced at the transducer was within its measurement range. $G(v)$ was measured over 4.5 decades in frequency $\left(3 \times 10^{-4} \mathrm{~Hz}<v<\right.$ $16 \mathrm{~Hz}$ ). The viscosity, which for tricresyl phosphate we measured over 11 decades, can be determined under both dc and ac conditions: under conditions of constant strain rate,

$$
\eta=\lim _{\dot{\gamma} \rightarrow 0}[\sigma(\dot{\gamma}) / \dot{\gamma}]
$$

where $\dot{\gamma} \equiv \mathrm{d} \gamma / \mathrm{d} t$; and as the limit of the low-frequency shear modulus,

$$
\eta=\lim _{v \rightarrow 0} \frac{G(v)}{2 \pi v}
$$



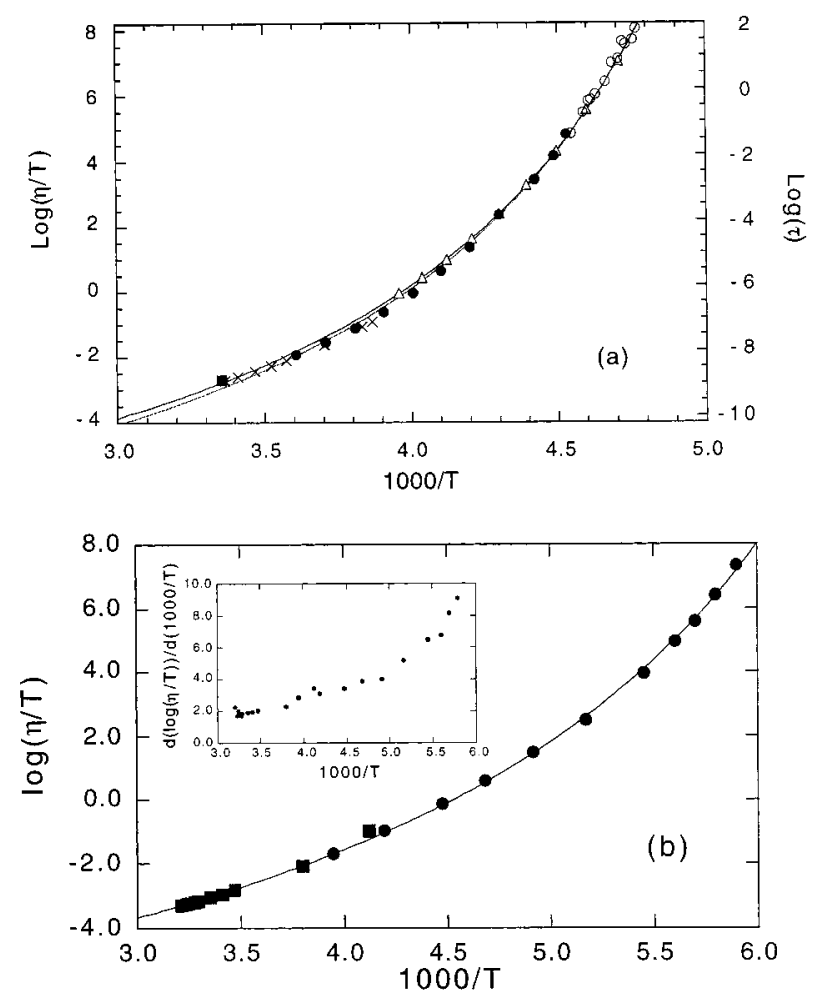

Figure 1. (a) Comparison of the temperature dependence of the viscosity and dielectric relaxation time for tricresyl phosphate. The left axis shows $\log _{10}\left(\eta\right.$ [poise]/T[Kelvin]), and the right axis shows $\log _{10} \tau$ [second] for the dielectric data. The solid and open circles are data for $\eta / T$ determined by the dc and ac methods, respectively, and the square was a point determined with a capillary tube viscometer. The crosses $(\times)$ are data from Barlow ${ }^{12}$ on a sample with a different isomeric content. The triangles $(\triangle)$ are data for $\tau$. The solid lines are the best Vogel-Fulcher fits to the data. For $\eta / T$ the fit is $\eta / T=7.1 \times 10^{-9}$ $\exp [1559 /(T-168)]$. For the relaxation time the fit is $\tau=8.6 \times 10^{-15}$ $\exp [1521 /(T-168)]$. The left- and right-hand axes have been shifted with respect to each other so that the data coincide in the upper righthand corner. (b) Plot of $\log _{10}(\eta / T)$ vs $1000 / T$ for squalane. The circles are our data measured by the dc method, and the squares are data taken from Barlow et al. ${ }^{12}$ The curve is a best fit of the Vogel-Fulcher form: $\eta / T=\left(1.0 \times 10^{-7}\right) \exp [1639 /(T-119)]$. The inset shows the derivative of $\log _{10}(\eta / T)$ with respect to $1000 / T$, indicating that there is no temperature at which the viscosity reverts to Arrhenius behavior.

To measure accurately the magnitude of $G(v)$ and $\eta$, one must calibrate the spacing between the plates precisely. Owing to thermal contraction and expansion of the rods holding the plates, this spacing changes with temperature and must be separately measured at each value of $T$. (However, to get the shape of the $G(v)$ curve-but not its magnitude-as a function of frequency, such a measurement is not necessary. By concentrating on the relative shape of the response curves rather than its magnitude, we are able to obtain good and reproducible data over a wide temperature range in a relatively short time.) Our technique for measuring the dielectric response has been presented elsewhere. ${ }^{11}$

Figure 1 shows the temperature dependence of the viscosity for the two liquids. In Figure 1a, the viscosity data for tricresyl phosphate is presented and compared with data on the same sample of the relaxation time measured by dielectric response. This relaxation time is found from the frequency of the peak, $\nu_{\mathrm{p}}$, in the imaginary part of the dielectric response: $\tau \equiv 1 /\left(2 \pi \nu_{\mathrm{p}}\right)$. For purposes of comparison with eq 1 , we plot $\log _{10} \eta / T$ on the left axis and $\log _{10} \tau$ on the right axis. The horizontal axis is $1000 / T$. The open and closed circles show $\eta / T$ determined by the dc and ac methods, respectively, and the triangles show the data for the relaxation time, $\tau$. The data by Barlow ${ }^{12}$ (on a sample with a different stated isomeric content) are shown by the crosses. The left and right axes are shifted so that the data coincide in the upper right-hand corner, allowing a direct comparison of the viscosity and the dielectric relaxation time over the entire temperature range covered. The data do not fall on a straight line on this graph (which would indicate Arrhenius or activated behavior) but is significantly curved. The upper solid line shows a Vogel-Fulcher fit to the relaxation time data: ${ }^{1}$

$$
\tau=\tau_{o} \exp \left[A /\left(T-T_{\mathrm{o}}\right)\right]
$$

The lower line is a similar fit for $\eta / T$. The Vogel-Fulcher fits are good in both cases. The values of $T_{\mathrm{o}}$ are the same within experimental error of one another for the two quantities.

In Figure $1 b$, we show a similar plot for $\log _{10} \eta / T$ versus $1000 / T$ for squalane. The circles are our data for $\eta / T$ measured by the dc method, and the squares are data taken from Barlow et al. ${ }^{12}$ These data are also well fit by a Vogel-Fulcher form. In the inset we show the derivative of the curve in order to see if there is a reversion to an Arrhenius form at low temperatures as has been suggested by mode-coupling theories. The data in the inset are monotonically increasing, which is inconsistent with Arrhenius behavior.

In Figure 2, we show the data for both the real and imaginary part of $G(v)=G^{\prime}(v)+\mathrm{i} G^{\prime \prime}(v)$ for tricresyl phosphate. At low frequencies, $G^{\prime}(v)$ varies quadratically with $v$, and at high frequencies, it approaches its high-frequency asymptote, $G_{\infty}$. At low frequencies, $G^{\prime \prime}(v)$ varies linearly, reaches a peak at frequency $v_{\mathrm{p}}$, and then decreases. The lines are fits to the data of the empirical Cole-Davidson form. The form we use is modified to be applicable for a modulus representation:

$$
G_{\mathrm{CD}}(v)=G_{\infty}\left(1-\frac{1}{\left(1+\mathrm{i} 2 \pi \nu \tau_{\mathrm{CD}}\right)^{\beta}}\right)
$$

For $\beta=1$ this reduces to the Maxwell model. ${ }^{3}$ In the ColeDavidson form, $G^{\prime \prime}(v) \propto v^{-\beta}$ above $\nu_{\mathrm{p}}$ and the smaller the value $\beta$, the wider will be the peak in $G^{\prime \prime}(v)$. In all cases this form fits our data very well but with an exponent $\beta$ significantly less than 1 . We note ${ }^{9}$ that $\tau_{\mathrm{CD}}$ is related to $\nu_{\mathrm{p}}$ by

$$
\tau_{\mathrm{CD}}=\frac{1}{2 \pi \nu_{\mathrm{p}}} \tan \left[\frac{\pi}{2(1+\beta)}\right]
$$

In Figure 3, we plot the best-fit value of the Cole-Davidson fitting parameter, $\beta$, versus temperature for tricresyl phosphate (circles) and squalane (squares). To capitalize on the greater strength of the $G^{\prime}(v)$ signal at frequencies above the peak of $G^{\prime \prime}(v)$ and of the $G^{\prime \prime}(v)$ signal at frequencies below the peak, a hybrid method is used to extract the characteristic values: $\nu_{\mathrm{p}}$, $G_{\infty}$, and $\beta$. First, $G^{\prime}(v)$ is fit to the real part of the ColeDavidson form (a three-parameter fit) given in eq 4 . The value of $G_{\infty}$ from this fit is then used as a constraint in fitting $G^{\prime \prime}(v)$ to the imaginary part of eq 4 (a two-parameter fit). The error bars on the high peak-frequency points are large because the peak of the spectrum is just entering our frequency window so that there is only a small portion of the high-frequency tail in the spectrum with which we can fit to the Cole-Davidson form. Our determination of $\beta$ in this region is governed by the shape of the spectrum near the peak. For both liquids, the value of $\beta$ increases as the peak frequency (and the temperature) decreases. This indicates that the shear modulus curves are becoming narrower as the glass transition is approached from above. This 

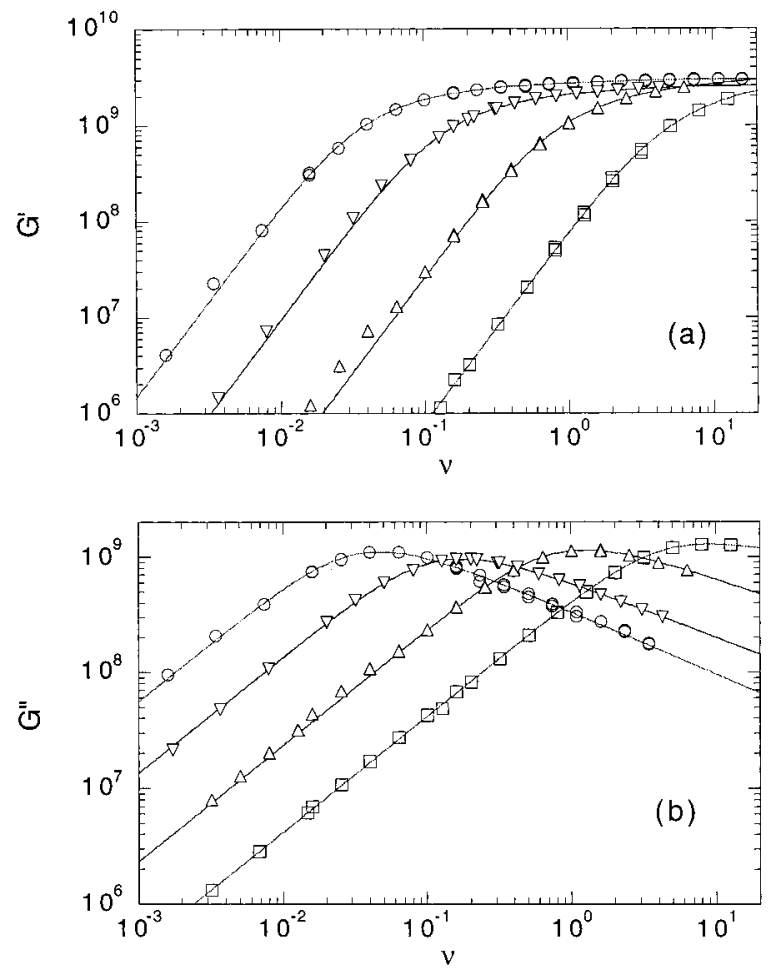

Figure 2. Real (a) and imaginary (b) part of $G(v)=G^{\prime}(v)+\mathrm{i} G^{\prime \prime}(v)$ for tricresyl phosphate. The circles $(O)$, inverted triangles $(\nabla)$, triangles $(\triangle)$, and squares $(\square)$ are for $T=211.8,213.3,215.1$, and $217.9 \mathrm{~K}$, respectively.

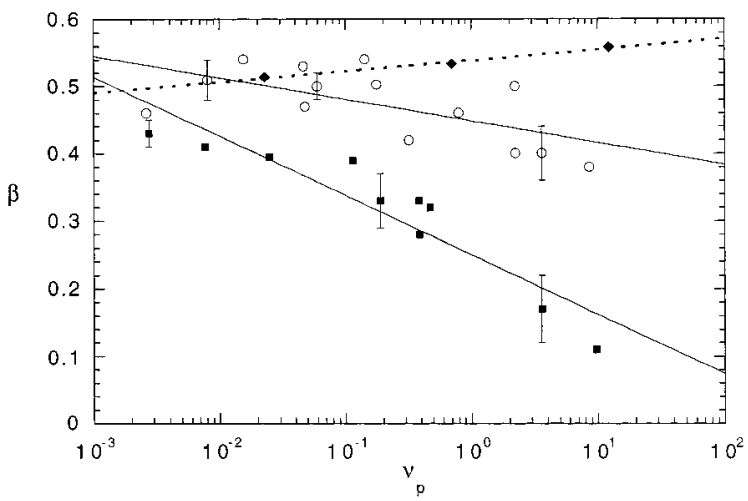

Figure 3. Exponent $\beta$ in the Cole-Davidson fitting form vs the peak frequency $v_{\mathrm{p}}$ for the shear modulus spectra of tricresyl phosphate $(O)$ and squalane (回) and for the dielectric spectra of tricresyl phosphate $(\diamond)$. The error bars on the high peak-frequency points are large because the peak of the spectrum is just entering our frequency window. Thus, for that data, there is only a small portion of the high-frequency tail in the spectrum that can be used to fit with the Cole-Davidson form. The straight lines through the data are meant as guides for the eye to emphasize the narrowing of $G^{\prime \prime}$ with decreasing temperature.

is the same trend as was found in the shear modulus of di-nbutylphthalate. ${ }^{9}$ We also show in the figure the value of $\beta$ obtained for the dielectric response for tricresyl phosphate, which has the opposite trend and decreases with decreasing temperature. We note, however, that the temperature dependence observed in the dielectric data is abnormally small compared with that found for many other nonionic supercooled liquids. ${ }^{13}$ (Despite the presence of an extremely small Johari-Goldstein $\beta$-relaxation in the high-frequency tail, the dielectric data for tricresyl phosphate can nevertheless be fit onto the same master curve as those other nonionic supercooled liquids.)

The increase in width of the response with increasing temperature that we find in the shear modulus data is very
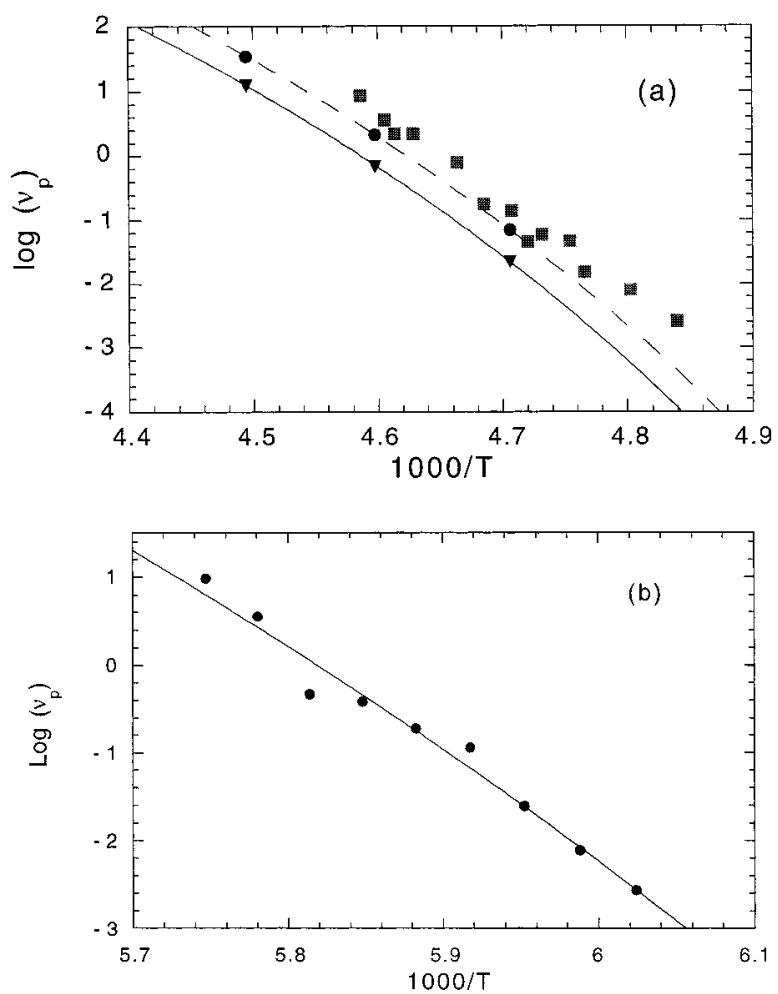

Figure 4. (a) Values of $v_{\mathrm{p}}$ determined from the shear modulus and the dielectric data as a function of $1000 / T$ for tricresyl phosphate. The squares ( $\square$ ) show the values of $v_{\mathrm{p}}$ taken from the peak positions of $G^{\prime \prime}(v)$. The triangles and solid line show the position of $\nu_{\mathrm{p}}$ taken from the dielectric data. The circles and dashed line show the shifted position of the peaks in the dielectric data, $v_{\mathrm{p}} \epsilon_{o} / \epsilon_{\infty}$, when converted to a modulus representation. (b) Values of $v_{\mathrm{p}}$ determined from the shear modulus as a function of $1000 / T$ for squalane.

different from what has been found in many other relaxation probes of nonionic glasses, such as the dielectric response or specific-heat spectroscopy. ${ }^{14}$ In ionic glasses, however, the electric modulus does have the same trend as we find here for the shear modulus. ${ }^{15-17}$ The electrical response of ionic glasses has a pole in the dissipative part of the dielectric susceptibility at $v=0$. This is also the case for the shear compliance of liquids: $-\mathrm{i} G^{\prime \prime}(v) /\left\{\left[G^{\prime}(v)\right]^{2}+\left[G^{\prime \prime}(v)\right]^{2}\right\}$ diverges as $v$ approaches zero. The presence of a pole at zero frequency in the compliance implies the existence of a nonzero transport coefficient (such as conductivity in the case of the electrical compliance and viscosity in the case of shear modulus). We suggest that the presence of this pole may be related to the difference between the temperature dependence for these two types of response; there is a correlation between whether the peaks get narrower or broader as the temperature is lowered and whether the susceptibility diverges or remains finite at zero frequency.

From the shear modulus data (as shown in Figure 2b) we can determine the variation of $v_{\mathrm{p}}$ on temperature. This is shown in Figure 4a for tricresyl phosphate and Figure $4 \mathrm{~b}$ for squalane. This frequency can be compared directly with the equivalent quantity determined from the dielectric response. This is shown for tricresyl phosphate in Figure 4a where the squares represent $v_{\mathrm{p}}$ obtained from the shear modulus data, $G^{\prime \prime}(v)$, and the triangles with the smooth curve show $v_{\mathrm{p}}$ obtained from the dielectric data, $\epsilon^{\prime \prime}(v)$. The dielectric susceptibility, $\epsilon(v)$, is the electrical compliance rather than the electrical modulus: $M(v) \equiv 1 / \epsilon(v)$. To get a valid comparison between the dielectric and the shear data, we must use values of $v_{\mathrm{p}}$ that both correspond to the appropriate response moduli. This entails shifting the dielectric data by a factor that is approximately the ratio of the zero- 


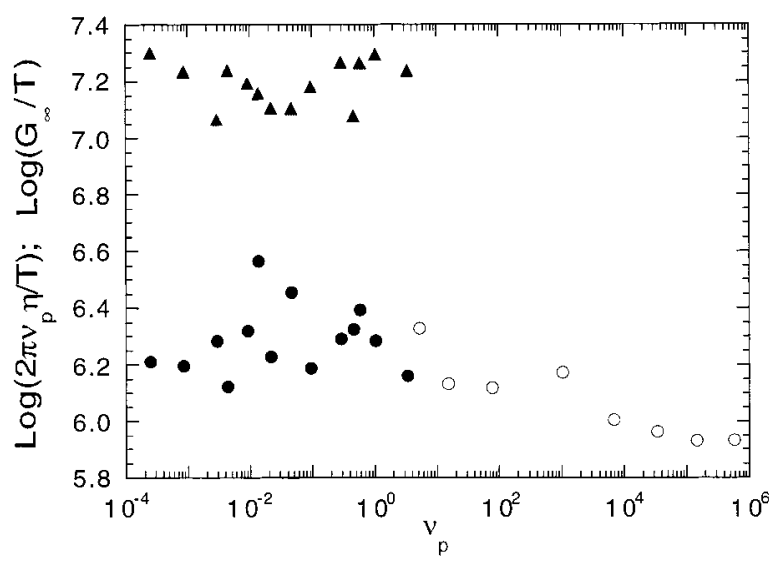

Figure 5. Ratios $R \equiv 2 \pi v_{\mathrm{p}} \eta / T$ and $G_{\infty} / T$ vs $v_{\mathrm{p}}$ for tricresyl phosphate. The triangles are for $G_{\infty} / T$; the solid and open circles are for values of $R$ computed using the viscosity measured by the ac and dc methods, respectively.

frequency to the infinite-frequency dielectric response: $\epsilon_{o} / \epsilon_{\infty}$. This can be seen by realizing that if the response is a simple Debye relaxation,

$$
\epsilon(v)=\mathrm{e}_{\infty}+\frac{\epsilon_{\mathrm{o}}-\epsilon_{\infty}}{1+\mathrm{i} 2 \pi \nu \tau_{\epsilon}}
$$

then

$$
M(v) \equiv \frac{1}{\epsilon}=M_{\mathrm{o}}-\frac{\left(M_{\mathrm{o}}-M_{\infty}\right) \mathrm{i} 2 \pi v \tau_{M}}{1+\mathrm{i} 2 \pi \nu \tau_{M}}
$$

where $M_{\mathrm{o}}=1 / \epsilon_{\mathrm{o}}, M_{\infty}=1 / \epsilon_{\infty}$, and $\tau_{M}=\tau_{\epsilon} \epsilon_{\infty} / \epsilon_{\mathrm{o}}$. The imaginary part of $M(v)$ thus also has the Debye form with a peak shifted by $\epsilon_{\mathrm{o}} / \epsilon_{\infty}$ from that in $\epsilon(v)$. For more complicated response functions, such as those found here with a Cole-Davidson form, this is the approximate shift factor that should be applied as long as the width of the response is not too much broader than that of Debye relaxation. ${ }^{18}$ The values of the peak frequency shifted by this factor is shown in Figure 4a by the solid circles with the dashed line. As we can see, the temperature dependence of the peak frequencies is very similar for the shear modulus and the dielectric data.

We now return to the question of whether there is any decoupling of the time scales as measured by the shear modulus and viscosity from that measured by the dielectric response in tricresyl phosphate. As we have just noted above, the data of Figure $4 \mathrm{a}$ show that the peak frequencies measured by the shear modulus have a value and temperature dependence very similar to the ones measured by dielectric response. This indicates that the shear modulus and dielectric measurements couple to the same set of underlying time scales and that there is no strong decoupling of the time scales as measured by these two probes. However, the data of Figure 1a does show a small systematic deviation between $\eta / T$ and the dielectric relaxation time $\tau$ when measured over a wide temperature range. Such deviations have, in the past, been used to imply a deviation from Einstein-Debye behavior as the glass transition temperature is approached..$^{-7}$ Following the earlier analysis of ref 9, we plot the ratio $R \equiv$ $2 \pi v_{\mathrm{p}} \eta / T$ versus $v_{\mathrm{p}}$ in Figure 5 with $v_{\mathrm{p}}$ obtained from the dielectric response. This ratio $R\left(T, v_{\mathrm{p}}\right)$ varies by at most only a factor of 3.5 as the peak frequency is varied by close to 10 orders of magnitude.

There are two circumstances in which substantially larger deviations from Stokes-Einstein behavior are seen. The first is in the conduction of small ionic species through glassy matrices with very large viscosities. The ionic mobility may decouple to an arbitrarily large extent from the viscosity in the host. Since this type of decoupling is not connected with the structural relaxation of the glass, it is not pertinent to the present discussion. The second example of large Stokes-Einstein deviations is found in comparisons of translational and rotational diffusion of probe molecules in supercooled liquids. ${ }^{19,20}$ Rotational diffusion times follow the temperature dependence of eq 1 with deviations comparable to those that we report; however, translational diffusion can become up to a factor of 100 faster at $T_{\mathrm{g}}$ than the Stokes-Einstein prediction. This rotationtranslation decoupling has been explained ${ }^{19-22}$ in terms of heterogeneous dynamics in the sample. It has been argued that rotational measurements yield an average over local relaxation times similar to that performed by viscosity and dielectric measurements, whereas the diffusion constant emphasizes regions where dynamics is faster than the average.

In Figure 5, we also plot for comparison the values of $G_{\infty} / T$. We note that in the temperature range measured, the value of $R$ does not coincide with the measured values of $G_{\infty} / T$. As the temperature (and $v_{\mathrm{p}}$ ) is decreased, the two values do approach each other. This effect is less pronounced here than it was for di- $n$-butylphthalate. ${ }^{9}$

As discussed in ref 9 a variation with temperature in the shape of $G^{\prime \prime}(v)$ may cause a small dependence of $R$ on $\nu_{\mathrm{p}}$ (or temperature) and a disparity between $R$ and $G_{\infty} / T$. The argument is that the Maxwell model (eq 2) assumes a single relaxation time, whereas the data show that there is a distribution of such times in the shear modulus that varies with temperature. As the temperature is lowered, the shear modulus becomes narrower, whereas the dielectric spectrum broadens. ${ }^{23}$ A model calculation based on the Cole-Davidson form for the shear modulus of eq 4 showed how a change in the value of $\beta$, like that shown in Figure 3, could alter the relative values of $\eta, G_{\infty}, \tau$, and $\nu_{\mathrm{p}}$ A combination of the increase in $\eta / \tau$ (obtained from shear modulus data) and $v_{\mathrm{p}} \tau$ (obtained from dielectric data) as temperature was lowered was sufficient to account for the observed variations in $R$. Thus, the small observed deviations from the Einstein relation could be explained in terms of a variation in the shape of the two spectra (shear modulus and dielectric) with temperature. The connection between the temperature dependence of the relaxation spectrum that we observe and the proposed growth of regions of heterogeneous dynamics ${ }^{19-22}$ remains unknown. Since all the moduli that display a narrowing of the shear modulus with decreasing temperature also have a zero-frequency pole in the imaginary part of the compliance, i.e., there is a transport coefficient associated with the modulus, we speculate that the experimentally observed separation of the translational and rotational diffusion coefficients and the narrowing of the modulus width are related.

Our new data on tricresyl phosphate likewise do not support a correlation length (extracted either from $R \equiv 2 \pi v_{\mathrm{p}} \eta / T$ or from $\left.G_{\infty} / T\right)$ increasing in the supercooled liquid as was suggested for $o$-terphenyl. ${ }^{7}$ Tricresyl phosphate and squalane have very similar behavior as was reported earlier ${ }^{9}$ on di- $n$-butylphthalate. A comment ${ }^{24}$ on that earlier paper had asserted that there was time-temperature superposition in that liquid and that there was no temperature dependence of the shape as had been claimed. The results reported here on two different liquids are consistent with the original data ${ }^{9,25}$ in that they do find a strong temperature dependence of the shape of the spectra for both liquids and thus corroborate the conclusion that time-temperature superposition is not a valid assumption for supercooled liquids approaching 
their glass transition. These new data on tricresyl phosphate also imply that there is no strong decoupling between the shear modulus relaxation times and those derived from probes such as the dielectric susceptibility.

Acknowledgment. We thank Austen Angell for his leadership in this field and for many inspiring discussions over the years, which have clarified our thinking, given us an overview of the field, and continually pointed us in new and fruitful directions for research on the glass transition. Funding was provided by NSF-DMR 9722646.

\section{References and Notes}

(1) For a review see the following. Ediger, M. D.; Angell, C. A.; Nagel, S. R. J. Phys. Chem. 1996, 100, 13200.

(2) Einstein, A. Ann. Phys. (N.Y.) 1905, 17, 549. 1980.

(4) Angell, C. A. J. Phys. Chem. Solids 1988, 49, 863

(5) Rössler, E. Phys. Rev. Lett. 1990, 65, 1595.

(6) Richter, D.; Zorn, R.; Farago, B.; Frick, B.; Fetters, L. J. Phys. Rev. Lett. 1992, 68, 71 .

(7) Fischer, E. W.; Donth, E.; Steffen, W. Phys. Rev. Lett. 1992, 68, 2344.

(8) See the following for a review of previous high-frequency $G(v)$ measurements. Lamb, J. Rheol. Acta 1973, 12, 438. Jeong, Y. H. Phys. $\operatorname{Rev}$. A 1987, 36, 766. Measurements made in a regime comparable to that of our data, but in the time domain, are detailed in the following: Plazek,
D. J.; Magill, J. H. J. Chem. Phys. 1966, 45, 3038; 1968, 49, 3678; Plazek, D. J.; Bero, C. A.; Chay, I.-C. J. Non-Cryst. Solids 1994, 172-174, 181. (9) Menon, N.; Nagel, S. R.; Venerus, D. C. Phys. Rev. Lett. 1994, 73,963 .

(10) Angell, C. A. In Proceedings of the Workshop on Relaxations in Complex Systems; Ngai, K. L., Wright, G. B., Eds.; National Technical Information Service, U.S. Department of Commerce: Springfield, VA, 1984; p 3.

(11) Deegan, R. D.; Nagel, S. R. Phys. Rev. B 1995, 52, 5653.

(12) Barlow, A. J.; Erginsav, A. Proc. R. Soc. London, Ser. A 1972, $327,175$.

(13) Dixon, P. K.; Wu, L.; Nagel, S. R.; Williams, B. D.; Carini, J. P. Phys. Rev. Lett. 1990, 65, 1108.

(14) Dixon, P. K.; Nagel, S. R. Phys. Rev. Lett. 1988, 61, 341.

(15) See, for example, the following. Howell, F. S.; Bose, R. A.; Macedo,

P. B.; Moynihan, C. T. J. Phys. Chem. 1974, 78, 639

(16) Hodge, I. M.; Angell, C. A. J. Chem. Phys. 1977, 67, 1647.

(17) Hasz, W. C.; Moynihan, C. T.; Tick, P. A. J. Non-Cryst. Solids

1994, 172-174, 1363.

(18) Jeong, Y. H.; Nagel, S. R.; Bhattacharya, S. Phys. Rev. A 1986 34,602 .

(19) Chang, I.; Sillescu, H. J. Phys. Chem. B 1997, 101, 8794. Fujara, F.; Geil, B.; Sillescu, H.; Fleischer, G. Z. Phys. B 1992, 88, 195.

(20) Cicerone, M. T.; Blackburn, F. R.; Ediger, M. D. J. Chem. Phys. 1995, 102, 471. Cicerone, M. T.; Ediger, M. D. J. Chem. Phys. 1996, 104, 7210.

(21) Stillinger, F. H.; Hodgdon, J. A. Phys. Rev. 1994, 50, 2064

(22) Tarjus, G.; Kivelson, D. J. Chem. Phys. 1995, 103, 3071.

(23) Leheny, R. L.; Nagel, S. R. Europhys. Lett. 1997, 39, 447.

(24) Behrens, C. F.; Christiansen, T. G.; Christensen, T.; Dyre, J. C.; Olsen, N. B. Phys. Rev. Lett. 1996, 76, 1553.

(25) Menon, N.; Nagel, S. R.; Venerus, D. C. Phys. Rev. Lett. 1996, 76,1554 . 